\title{
Résumé de la mise à jour du CCNI sur l'immunisation durant la grossesse avec le vaccin combiné anti-Tétanos, et à dose réduite contre la diphtérie et la coqueluche acellulaire (dcaT)
}

\author{
J Brophy' ${ }^{1}$ O Baclic², MC Tunis ${ }^{2}$ au nom du Comité consultatif national de l'immunisation (CCNI)*
}

\section{Résumé}

Contexte : La coqueluche est une maladie endémique cyclique au Canada qui connaît des pics $\mathrm{d}^{\prime}$ activité tous les deux à cinq ans. Bien que l'incidence de la coqueluche varie selon le groupe d'âge, les nourrissons non immunisés ou sous-immunisés sont les plus à risque d'infection et de complications connexes. Depuis les dernières recommandations du Comité consultatif national de l'immunisation publiées en 2014, de nouvelles données probantes nous renseignent sur l'innocuité et l'efficacité du vaccin combiné anti-Tétanos, et à dose réduite contre la diphtérie et la coqueluche acellulaire (dcaT) administré durant la grossesse.

Objectif : Fournir une orientation sur la vaccination maternelle pendant la grossesse comme stratégie visant à réduire l'incidence de la maladie et ses répercussions graves (comme I'hospitalisation ou la mort) découlant d'une infection par la coqueluche chez les nourrissons de moins de 12 mois.

Méthodes : Le CCNI a examiné les données probantes sur le fardeau de la maladie au Canada et sur l'innocuité, l'immunogénicité et l'efficacité du vaccin dans des administrations qui ont mis en œuvre des programmes de vaccination maternelle. Au total, 59 articles ont été recensés, extraits et mentionnés dans la revue de la littérature afin de documenter la déclaration.

Résultats : Dans la majorité des études examinées, les taux d'anticorps ont augmenté après l'immunisation, donnant lieu chez plus de $90 \%$ des femmes à des taux d'anticorps anti-PT supérieurs ou égaux à $10 \mathrm{UI} / \mathrm{ml}$, un mois après l'immunisation. Chez les nourrissons, vaccination maternellea eu pour effet d'augmenter les concentrations d'anticorps anticoquelucheux. Dans la majorité des études, l'administration de la quatrième dose du vaccin contre la diphtérie, la coqueluche acellulaire et le tétanos (DCaT) à l'âge de 15 mois n'a révélé aucune différence statistiquement significative dans les taux d'anticorps et dans l'avidité entre les nourrissons dont la mère a reçu le vaccin dcaT durant la grossesse et ceux dont la mère ne l'avait pas reçu durant la grossesse. La revue de la littérature ne rapporte aucun problème d'innocuité pour la mère ou le nourrisson, ni pour l'issue de la grossesse. On estime que le vaccin dcaT administré à la mère en cours de grossesse est efficace à plus de $90 \%$ contre la coqueluche chez les nourrissons de moins de deux mois. Aucun décès n'a été observé chez les nourrissons dont la mère a reçu le vaccin dcaT avant la $36^{\circ}$ semaine de grossesse. L'administration du vaccin dcaT chez les mères pendant la grossesse a également entraîné une réduction de maladies graves et d'hospitalisation chez le nourrisson. L'efficacité du vaccin s'est également révélée durable après l'administration des trois premières doses du vaccin $\mathrm{DCaT}$, procurant une protection supplémentaire atteignant $70 \%$ chez les enfants dont la mère a reçu le vaccin dcaT durant la grossesse.

Conclusion : Il existe maintenant une preuve solide à l'appui de la recommandation du CCNI selon laquelle l'immunisation à l'aide du vaccin dcaT doit être offerte systématiquement à la grossesse. Le vaccin doit être idéalement administré entre la $27^{\text {e }}$ et la $32^{\mathrm{e}}$ semaine de grossesse, mais des données probantes appuient aussi l'administration du vaccin dans un spectre plus large d'âges gestationnels. Ainsi, selon l'état particulier de la patiente et d'autres facteurs programmatiques, il pourrait être offert à partir de la $13^{e}$ semaine jusqu'au moment de l'accouchement.

\section{Affiliations}

${ }^{1}$ Centre hospitalier pour enfants de l'est de l'Ontario (Ontario)

${ }^{2}$ Centre de l'immunisation et des maladies respiratoires infectieuses, Agence de la santé publique du Canada, Ottawa (Ontario)

*Correspondance : naci-ccni@ phac-aspc.gc.ca
Citation proposée : Brophy J, Baclic O, Tunis MC au nom du Comité consultatif national de l'immunisation (CCNI). Résumé de la mise à jour du CCNI sur l'immunisation durant la grossesse à l'aide d'un vaccin combiné anti-Tétanos, et à dose réduite contre la diphtérie et la coqueluche acellulaire (dcaT). Relevé des maladies transmissibles au Canada. 2018;44(3/4):101-5. https://doi.org/10.14745/ccdr.v44i34a04f 


\section{Introduction}

La coqueluche est une maladie endémique cyclique au Canada qui connaît des pics d'activité tous les deux à cinq ans. Bien que l'incidence de la coqueluche varie selon le groupe d'âge, les nourrissons non immunisés ou sous-immunisés sont les plus à risque d'infection et de complications connexes. Entre 2006 et 2015, les taux d'incidence et d'hospitalisation moyens propres à l'âge étaient plus élevés chez les nourrissons de moins d'un an (71,2 et 33,6 cas par 100000 habitants). Entre 2006 et 2016, les nourrissons de moins de deux mois représentaient la plus grande proportion des admissions aux unités spécialisées (40,5\%), suivis des nourrissons de trois à quatre mois $(21,4 \%)$.

L'absence de vaccination maternelle devrait accroître la susceptibilité du nourrisson à l'infection, puisqu'elle augmente le risque de maladie chez la mère (et sa transmission ultérieure au nourrisson). L'absence d'immunisation ne fournit pas non plus une protection passive suffisante par le passage transplacentaire d'anticorps ou par le lait maternel. Une récente étude sérologique a révélé que chez la majorité des femmes enceintes au Canada (plus de $90 \%$ ), les niveaux d'anatoxine coquelucheuse étaient indétectables. Dans une étude menée en 2017 sur les pratiques d'immunisation au Canada, la majorité des gouvernements provinciaux et territoriaux ont signalé des difficultés d'ordre logistique à mettre en œuvre des programmes d'immunisation chez les mères à l'aide d'un vaccin combiné anti-Tétanos, et à dose réduite contre la diphtérie et la coqueluche acellulaire (dcaT) en situation d'éclosion seulement.

Depuis les dernières recommandations du CCNI publiées en 2014, de nouvelles données probantes nous renseignent sur l'innocuité et l'efficacité du vaccin dcaT administré durant la grossesse. En accord avec les orientations fournies par le Comité canadien sur l'immunisation, le CCNI a examiné les aspects suivants :

- le fardeau de la coqueluche chez les nourrissons de moins de 12 mois;

- l'innocuité de la vaccination maternelle à l'aide du vaccin dcaT durant la grossesse;

- l'efficacité de la vaccination maternelle à l'aide du vaccin dcaT pendant la grossesse afin de prévenir des complications graves découlant d'une infection par la coqueluche chez les nourrissons de moins de 12 mois;

- les effets de la vaccination maternelle à l'aide du vaccin dcaT durant la grossesse sur la réaction immunologique du nourrisson dans le cadre du programme initial de vaccination;

- I'incidence de la vaccination maternelle à l'aide du vaccin dcaT durant la grossesse sur la protection à long terme contre la coqueluche chez les enfants.

La mise à jour de la déclaration a pour objectif de fournir une orientation sur la vaccination maternelle pendant la grossesse comme stratégie visant à réduire l'incidence de la maladie et ses répercussions graves (comme l'hospitalisation ou la mort) découlant d'une infection par la coqueluche chez les nourrissons de moins de 12 mois. La mise à jour complète est accessible en ligne (1). Cet article est un résumé des principales conclusions de la mise à jour.

\section{Méthodes}

Le CCNI a examiné les données probantes sur le fardeau de la maladie au Canada et sur l'innocuité, l'immunogénicité et l'efficacité du vaccin dans des administrations qui ont mis en $œ u v r e$ des programmes de vaccination maternelle. Au total, 59 articles ont été recensés, extraits et mentionnés dans la revue de la littérature afin de documenter la déclaration. L'analyse épidémiologique a été réalisée à l'aide des données de surveillance nationales, notamment le Système canadien de surveillance des maladies à déclaration obligatoire (SCSMDO), le programme canadien de surveillance active de l'immunisation (IMPACT) et la Base de données sur les congés des patients (BDCP) de I'Institut canadien d'information sur la santé. Les données probantes relatives aux questions suivantes ont fait l'objet d'un examen et d'une évaluation critique :

- Ya-t-il une différence significative dans les manifestations indésirables locales ou systémiques entre l'immunisation de femmes enceintes à l'aide du vaccin dcaT (à tous les stades) et l'immunisation de femmes non enceintes?

- $\quad$ a-t-il une différence significative dans les effets indésirables sur l'état de santé du fœtus et du nouveau-né après l'immunisation de la mère à l'aide du vaccin dcaT durant la grossesse?

- La vaccination maternelle pendant la grossesse à l'aide du vaccin dcaT est-elle significativement plus efficace pour la prévention de maladies graves chez les nourrissons de moins de 12 mois comparativement à l'absence d'une vaccination maternelle pendant la grossesse?

- L'immunogénicité du vaccin contre la diphtérie, la coqueluche acellulaire et le tétanos (DCaT) chez les enfants nés de mères immunisées à l'aide du vaccin dcaT durant la grossesse est-elle significativement différente comparativement aux nourrissons nés de mères qui n'ont pas été immunisées à l'aide du vaccin dcaT durant la grossesse?

- La vaccination maternelle à l'aide du vaccin dcaT pendant la grossesse influe-t-elle significativement sur l'efficacité du vaccin DCaT pour la prévention de maladies connexes chez les enfants de moins de quatre à six ans? Une analyse détaillée de la littérature a été publiée dans une revue distincte du CCNI (2). Une synthèse des données probantes et un résumé exhaustif de la littérature ainsi que des recommandations précises ont été fournis dans la mise à jour de la déclaration du CCNI (1).

\section{Résultats}

\section{Immunogénicité}

Dans la majorité des études examinées, les taux d'anticorps ont augmenté après l'immunisation, donnant lieu chez plus de $90 \%$ des femmes à des taux d'anticorps anti-PT supérieurs ou égaux à $10 \mathrm{UI} / \mathrm{ml}$, un mois après I'immunisation. Bien qu'aucune étude sérologique actuelle n'établit de corrélation avec une possible protection clinique contre la coqueluche, des taux d'anticorps anti-PT supérieurs ou égaux à $10 \mathrm{Ul} / \mathrm{ml}$ sont considérés comme protecteurs contre la maladie grave. Chez les nourrissons, la vaccination maternelle a eu pour effet d'augmenter les concentrations d'anticorps anticoquelucheux et d'augmenter 
I'avidité de manière linéaire jusqu'à l'accouchement. Dans la majorité des études, l'administration de la quatrième dose du vaccin contre la diphtérie, la coqueluche acellulaire et le tétanos (DCaT) à l'âge de 15 mois n'a révélé aucune différence statistiquement significative dans les taux d'anticorps et dans l'avidité entre les nourrissons dont la mère a reçu le vaccin dcaT durant la grossesse et ceux dont la mère ne l'avait pas reçu durant la grossesse.

\section{Innocuité et efficacité}

La revue de la littérature ne rapporte aucun problème d'innocuité pour la mère ou le nourrisson, ni pour l'issue de la grossesse. On estime que le vaccin dcaT administré à la mère en cours de grossesse est efficace à plus de $90 \%$ contre la coqueluche chez les nourrissons de moins de deux mois. Aucun décès n'a été observé chez les nourrissons dont la mère a reçu le vaccin dcaT avant la $36^{\mathrm{e}}$ semaine de grossesse. L'administration du vaccin dcaT chez les mères pendant la grossesse a également entraîné une réduction de maladies graves et d'hospitalisation chez le nourrisson. L'efficacité du vaccin s'est également révélée durable après l'administration des trois premières doses du vaccin DCaT. L'immunisation durant la grossesse a permis de réduire de $70 \%$ le risque que les enfants vaccinés, dont la mère a reçu le vaccin dcaT, contractent la coqueluche.

\section{Recommandations}

À la suite de l'examen des données probantes actuelles, le CCNI a formulé une recommandation pour introduire le vaccin dcaT dans le calendrier de vaccination systématique lors de chaque grossesse. Les détails complets de la revue de littérature, le justificatif et les facteurs pertinents des recommandations à jour figurent dans la mise à jour du CCNI en cette matière (1) et dans la revue de littérature du CCNI (2).

Le CCNI recommande que l'immunisation à l'aide du vaccin dcaT soit offerte systématiquement lors de chaque grossesse sans égard aux antécédents de vaccination au dcaT (forte recommandation du CCNI). Le CCNI conclut qu'il existe des preuves suffisantes pour recommander une telle immunisation (données probantes de catégorie A).

L'immunisation systématique des mères durant la grossesse à l'aide du vaccin dcaT fournira une protection plus robuste et plus complète contre la coqueluche chez les nourrissons comparativement à une immunisation en situation d'éclosion seulement. Il a été démontré que l'immunisation à l'aide du vaccin dcaT en cours de grossesse protège neuf nourrissons sur dix âgés de moins de trois mois contre la coqueluche. Aucun problème important d'innocuité n'a été décelé dans l'ensemble de la littérature scientifique actuelle et aucun risque accru de complications graves durant la grossesse pour la mère ou le nourrisson n'a été signalé dans les pays qui offrent systématiquement le vaccin dcaT pour l'immunisation à la grossesse. De même, aucune manifestation indésirable grave n'a été décelée au Canada par le Système canadien de surveillance des effets secondaires suivant l'immunisation (SCSESSI). II n'y a actuellement aucun indice de changement cliniquement important dans le déclenchement de la mémoire immunologique des nourrissons exposés à des concentrations accrues d'anticorps d'origine maternelle après une immunisation à l'aide du vaccin dcaT en cours de grossesse. Étant donné la rapide diminution des anticorps de la mère qui été observée dans les études, l'immunisation doit être offerte systématiquement à la grossesse, sans égard aux antécédents de vaccination ou à l'intervalle entre les grossesses.

Le CCNI recommande que l'immunisation à l'aide du vaccin dcaT soit idéalement offerte entre la $27^{\mathrm{e}}$ et la $32^{\mathrm{e}}$ semaine de grossesse (forte recommandation du CCNI selon les données probantes de catégorie A). Les données probantes soutiennent également le bien-fondé de l'administration du vaccin dcaT aux mères dans un spectre plus large d'âges gestationnels. Le CCNI recommande pour sa part qu'il soit offert à partir de la $13^{\mathrm{e}}$ semaine jusqu'à l'accouchement, selon l'état particulier de la patiente et d'autres facteurs programmatiques (recommandation à caractère discrétionnaire du CCNI selon les données probantes de catégorie $A / B)$.

$L^{\prime}$ immunisation devrait idéalement être offerte de la $27^{\mathrm{e}}$ à la $32^{\mathrm{e}}$ semaine de grossesse. Cette recommandation est appuyée par des données probantes qui indiquent que son innocuité et son efficacité y sont les meilleures. L'immunisation entre la $13^{\mathrm{e}}$ et la $26^{\mathrm{e}}$ semaine de grossesse peut également être envisagée dans certaines situations ( $p$. ex., lors d'une grossesse présentant un risque accru d'accouchement prématuré) afin de permettre une exposition placentaire hâtive à des taux d'anticorps plus élevés et une maximisation du passage transplacentaire des anticorps. Il est préférable que le vaccin soit administré dans un délai suffisamment long avant la naissance (c.-à-d. quatre semaines) afin de permettre un passage transplacentaire efficace des anticorps et la protection du nourrisson contre la coqueluche. Néanmoins, une telle immunisation devrait être envisagée jusqu'à la fin de la grossesse chez les femmes qui ne l'ont pas encore obtenue, car elle est susceptible de fournir minimalement une protection partielle. Si l'immunisation à l'aide du vaccin dcaT a eu lieu au début de la grossesse (p. ex., avant la reconnaissance de la grossesse), il n'est pas nécessaire de l'offrir à nouveau après la $13^{\mathrm{e}}$ semaine.

Divers choix de calendrier de vaccination contre la coqueluche sont possibles; la décision à savoir quel est le meilleur choix peut dépendre des facteurs détaillés dans le tableau 1 ci-dessous. 
Tableau 1 : Choix et facteurs pouvant intervenir dans l'immunisation contre la coqueluche pendant la grossesse

\begin{tabular}{|c|c|c|}
\hline Choix & Facteurs & Points de décisions \\
\hline $\begin{array}{l}\text { 1. Immunisation } \\
\text { de la } 27^{\mathrm{e}} \text { à la } \\
32^{\mathrm{e}} \text { semaine } \\
\text { de grossesse }\end{array}$ & $\begin{array}{l}\text { Innocuité } \\
\text { - Solides données sur } \\
\text { l'innocuité au troisième } \\
\text { trimestre de grossesse } \\
\text { Efficacité } \\
\text { - Les données sur } \\
\text { l'efficacité prolongent } \\
\text { essentiellement la durée } \\
\text { de la vaccination (de la } \\
27 \text { à la } 36^{e} \text { semaine de } \\
\text { grossesse) } \\
\text { Immunogénicité } \\
\text { - Les taux d'anticorps } \\
\text { anticoquelucheux chez } \\
\text { la mère atteignent leur } \\
\text { sommet environ quatre } \\
\text { semaines après la } \\
\text { vaccination } \\
\text { Le passage } \\
\text { transplacentaire des } \\
\text { anticorps de la mère } \\
\text { est optimal au troisième } \\
\text { trimestre de la grossesse } \\
\text { Faisabilité ou acceptabilité } \\
\text { Peut être jumelé à un } \\
\text { suivi prénatal courant } \\
\text { lors duquel un dépistage } \\
\text { du diabète de grossesse } \\
\text { est offert (de la } 24 \text { e à la } \\
28 \text { e semaine de grossesse) }\end{array}$ & $\begin{array}{l}\text { Équilibre optimal } \\
\text { entre les données } \\
\text { sur l'innocuité, les } \\
\text { possibilités cliniques, } \\
\text { la faible probabilité de } \\
\text { diminution des anticorps, } \\
\text { la formation et le } \\
\text { passage transplacentaire } \\
\text { efficaces des anticorps } \\
\text { pour les grossesses à } \\
\text { terme. } \\
\text { Ce choix est appuyé par } \\
\text { des données probantes } \\
\text { qui indiquent que son } \\
\text { innocuité et son efficacité } \\
\text { y sont les meilleures } \\
\text { parmi l'ensemble des } \\
\text { possibilités, et qu'il } \\
\text { laisse suffisamment } \\
\text { de temps à la réponse } \\
\text { anticorps pour se former } \\
\text { pleinement durant la } \\
\text { grossesse. } \\
\text { L'immunisation peut } \\
\text { être jumelée à un suivi } \\
\text { courant de la grossesse, } \\
\text { mais elle ne garantit pas } \\
\text { de fournir une protection } \\
\text { pour certaines naissances } \\
\text { prématurées. }\end{array}$ \\
\hline $\begin{array}{l}\text { 2. Immunisation } \\
\text { de la } 13^{\mathrm{e}} \text { à la } \\
26^{\mathrm{e}} \text { semaine } \\
\text { de grossesse }\end{array}$ & $\begin{array}{l}\text { Innocuité } \\
\text { Données sur l'innocuité } \\
\text { plus faibles au deuxième } \\
\text { trimestre de grossesse } \\
\text { Efficacité } \\
\text { - Données sur l'efficacité } \\
\text { non stratifiées pour une } \\
\text { immunisation au deuxième } \\
\text { trimestre de la grossesse } \\
\text { (comprend la vaccination } \\
\text { au deuxième et au } \\
\text { troisième trimestre de la } \\
\text { grossesse) } \\
\text { Immunogénicité } \\
\text { - Les taux d'anticorps } \\
\text { anticoquelucheux chez } \\
\text { la mère atteignent leur } \\
\text { sommet environ quatre } \\
\text { semaines après la } \\
\text { vaccination } \\
\text { Certaines études ont } \\
\text { montré une plus grande } \\
\text { concentration d'anticorps } \\
\text { chez les nourrissons } \\
\text { après une vaccination de } \\
\text { la } 13^{e} \text { à la } 25^{\mathrm{e}} \text { semaine } \\
\text { comparativement à celle } \\
\text { observée après une } \\
\text { vaccination à partir de la } \\
26 \text { semaine } \\
\text { - } \text { 'administration hâtive } \\
\text { du vaccin au deuxième } \\
\text { trimestre a donné lieu à } \\
\text { des anticorps de plus forte } \\
\text { avidité (affinité) } \\
\text { Faisabilité ou acceptabilité } \\
\text { Peut être jumelé avec un } \\
\text { suivi prénatal courant, } \\
\text { soit après l'examen d'une } \\
\text { échographie anatomique } \\
\text { détaillée (effectuée } \\
\text { généralement de la } \\
\text { 18e à la } 222^{\mathrm{e}} \text { semaine de } \\
\text { grossesse) ou lors du } \\
\text { dépistage du diabète de } \\
\text { grossesse (de la } 24 \text { è à la } \\
\text { 28e semaine de grossesse) }\end{array}$ & $\begin{array}{l}\text { Les données sur } \\
\text { l'innocuité sont plus } \\
\text { faibles au deuxieme } \\
\text { trimestre et les données } \\
\text { sur l'efficacité ne sont } \\
\text { pas stratifiées pour } \\
\text { une immunisation au } \\
\text { deuxième trimestre de } \\
\text { grossesse. } \\
\text { La vaccination au } \\
\text { deuxième trimestre } \\
\text { augmente les possibilités } \\
\text { cliniques d'offrir le } \\
\text { vaccin et assure la } \\
\text { formation et le passage } \\
\text { transplacentaire } \\
\text { optimaux d'anticorps } \\
\text { pour les nourrissons } \\
\text { et les prématurés. } \\
\text { Lors d'accouchements } \\
\text { prématurés, une faible } \\
\text { possibilité existe pour le } \\
\text { passage transplacentaire } \\
\text { d'anticorps entre } \\
\text { la } 28 \text { e semaine et } \\
\text { l'accouchement. }\end{array}$ \\
\hline $\begin{array}{l}\text { 3. Immunisation } \\
\text { avant la } \\
13^{\mathrm{e}} \text { semaine } \\
\text { de grossesse }\end{array}$ & $\begin{array}{l}\text { Innocuité } \\
\text { - Données limitées sur } \\
\text { l'innocuité au premier } \\
\text { trimestre }\end{array}$ & $\begin{array}{l}\text { Les données sur } \\
\text { l'innocuité sont limitées } \\
\text { avant la } 13^{\mathrm{e}} \text { semaine }\end{array}$ \\
\hline
\end{tabular}

Tableau 1 : Choix et facteurs pouvant intervenir dans l'immunisation contre la coqueluche pendant la grossesse (suite)

\begin{tabular}{|c|c|c|}
\hline Choix & Facteurs & Points de décisions \\
\hline $\begin{array}{l}\text { 3. Immunisation } \\
\text { avant la } \\
13^{\mathrm{e}} \text { semaine } \\
\text { de grossesse } \\
\text { (suite) }\end{array}$ & $\begin{array}{l}\text { Efficacité } \\
\text { - Aucune donnée sur } \\
\text { l'efficacité stratifiée pour } \\
\text { une immunisation avant la } \\
13^{e} \text { semaine de grossesse } \\
\text { Immunogénicité } \\
\text { - Les anticorps de la } \\
\text { mère vont commencer } \\
\text { à diminuer avant } \\
\text { l'accouchement à terme } \\
\text { - Le passage } \\
\text { transplacentaire des } \\
\text { anticorps de la mère est } \\
\text { minime avant le troisième } \\
\text { trimestre de grossesse } \\
\text { Faisabilité ou acceptabilité } \\
\text { Si le vaccin est administré } \\
\text { avant l'échographie } \\
\text { anatomique détaillée, } \\
\text { des anomalies fœetales et } \\
\text { d'autres complications } \\
\text { liées à la grossesse } \\
\text { pendant le premier } \\
\text { trimestre peuvent être } \\
\text { imputées à tort au vaccin } \\
\text { - Le vaccin peut ne pas } \\
\text { être considéré comme } \\
\text { valable par les patientes } \\
\text { et les cliniciens au premier } \\
\text { trimestre de la grossesse }\end{array}$ & $\begin{array}{l}\text { Lorsqu'ils sont } \\
\text { administrés au début } \\
\text { de la grossesse, les } \\
\text { anticorps peuvent } \\
\text { diminuer avant } \\
\text { l'accouchement à terme. } \\
\text { Il y a un risque que } \\
\text { des manifestations } \\
\text { indésirables liées à } \\
\text { la grossesse soient } \\
\text { imputées à tort au } \\
\text { vaccin. }\end{array}$ \\
\hline $\begin{array}{l}\text { 4. Immunisation } \\
\text { après la } \\
32^{\mathrm{e}} \text { semaine } \\
\text { de grossesse }\end{array}$ & $\begin{array}{l}\text { Innocuité } \\
\text { - Solides données sur } \\
\text { l'innocuité au troisième } \\
\text { trimestre de grossesse } \\
\text { Efficacité } \\
\text { - Les données sur } \\
\text { l'efficacité prolongent } \\
\text { essentiellement la durée } \\
\text { de la vaccination (de la } \\
27 \text { à la } 36 \text { e semaine de } \\
\text { grossesse) } \\
\text { Immunogénicité } \\
\text { - Le passage } \\
\text { transplacentaire des } \\
\text { anticorps de la mère } \\
\text { est optimal au troisième } \\
\text { trimestre de la grossesse } \\
\text { Les taux d'anticorps } \\
\text { anticoquelucheux chez } \\
\text { la mère atteignent leur } \\
\text { sommet environ quatre } \\
\text { semaines après la } \\
\text { vaccination } \\
\text { Faisabilité ou acceptabilité } \\
\text { - Les possibilités cliniques } \\
\text { de vaccination sont } \\
\text { élargies grâce au suivi } \\
\text { prénatal courant vers la fin } \\
\text { de la grossesse }\end{array}$ & $\begin{array}{l}\text { Les données sur } \\
\text { l'innocuité et l'efficacité } \\
\text { sont les plus solides } \\
\text { à partir du troisième } \\
\text { trimestre de grossesse. } \\
\text { Ce choix peut ne pas } \\
\text { laisser suffisamment de } \\
\text { temps (c.-à-d. quatre } \\
\text { semaines) pour la } \\
\text { formation et le passage } \\
\text { transplacentaire des } \\
\text { anticorps de la mère } \\
\text { avant l'accouchement. } \\
\text { L'immunisation tardive } \\
\text { n'assurera aucune } \\
\text { protection à la plupart } \\
\text { des prématurés. } \\
\text { Il peut ne pas exister } \\
\text { de possibilités cliniques } \\
\text { suffisantes pour offrir } \\
\text { la vaccination à la } \\
\text { fin de la grossesse } \\
\text { comparativement à une } \\
\text { vaccination hâtive. }\end{array}$ \\
\hline
\end{tabular}

\section{Conclusion}

Il existe maintenant une preuve solide à l'appui de la recommandation du CCNI selon laquelle l'immunisation à l'aide du vaccin dcaT doit être offerte systématiquement à la grossesse. Le vaccin doit être idéalement administré entre la $27^{e}$ et la $32^{e}$ semaine de grossesse, mais des données probantes appuient aussi l'administration du vaccin dans un spectre plus large d'âges gestationnels. Ainsi, selon l'état particulier de la patiente et d'autres facteurs programmatiques, il pourrait être offert à partir de la $13^{\text {e }}$ semaine jusqu'au moment de l'accouchement. 


\section{Déclaration des auteurs}

Cette déclaration a été préparée par le groupe de travail du CCNI constitué des membres suivants : $D^{r}$ J. Brophy (président), $D^{r}$ N. Brousseau, D ${ }^{\text {re }}$ E. Castillo, D ${ }^{\text {re }}$ N. Crowcroft, $D^{\text {re }}$ S. Deeks, Dr I. Gemmill, Dr S. Halperin, D' B. Henry, Dre M. Naus, $D^{r e}$ M. Salvadori et $D^{r}$ B. Seifert.

\section{Conflit d'intérêt}

Aucun.

\section{Remerciements}

Le CCNI reconnaît et apprécie la contribution de S. Duchesne-Bélanger (Centre de l'immunisation et des maladies respiratoires infectieuses [CIMRI], Agence de la santé publique du Canada [ASPC]), L. Gamble (Bibliothèque de la santé), J. Rotondo (CIMRI, ASPC), et M. Saboui (CIMRI, ASPC) pour la déclaration.

Membres du CCNI : Dre C. Quach (présidente), Dre W. Vaudry (vice-présidente), Dre N. Dayneka, DrP. DeWals, D ${ }^{\text {re }}$ S. Deeks, $D^{r e}$ V. Dubey, D ${ }^{r e}$ R. Harrison, Dr M. Lavoie, $D^{r}$ C. Rotstein, $D^{r e}$ M. Salvadori, $D^{r e} B$. Sander, D ${ }^{r e}$ N. Sicard et $D^{r}$ R. Warrington

Anciens membres du CCNI : D ${ }^{r e}$ B. Henry, Dr I. Gemmil, $D^{\text {re }}$ S. Marchant-Short et $D^{r} D$. Vinh

Agents de liaison : Dr J. Brophy (Association canadienne pour la recherche et l'évaluation en immunisation [CAIRE]), $D^{r e} E$. Castillo (Société des obstétriciens et gynécologues du Canada), Dre A. Cohn (Centers for Disease Control and Prevention, États-Unis), Mme T. Cole (Comité canadien sur I'immunisation), Dre J. Emili (Collège des médecins de famille du Canada), Dre K. Klein (Conseil des médecins hygiénistes en chef), $\mathrm{D}^{\text {re }} \mathrm{C}$. Mah (Association canadienne de santé publique), $D^{\text {re }} \mathrm{D}$. Moore (Société canadienne de pédiatrie), $\mathrm{D}^{\mathrm{re}}$ A. Pham-Huy
(Association pour la microbiologie médicale et l'infectiologie [AMMI] Canada)

Anciens agents de liaison : $D^{\text {re }} \mathrm{J}$. Blake (Société des obstétriciens et gynécologues du Canada)

Représentants d'office : Dre (Capc) K. Barnes (ministère de la Défense nationale et Forces armées canadiennes), $M^{\text {me }} \mathrm{G}$. Charos (CIMRI, ASPC), Dre G. Coleman (Direction des produits biologiques et des thérapies génétiques, Santé Canada [SC]), $D^{r}$ J. Gallivan (Direction des produits de santé commercialisés [DPSC], SC), M ${ }^{\text {me }}$ J. Pennock (CIMRI, ASPC), M. G. Poliquin (Laboratoire national de microbiologie), Dr T. Wong (Direction générale de la santé des Premières nations et des Inuits [DGSPNI], SC)

\section{Financement}

Les travaux du CCNI sont financés par l'Agence de la santé publique du Canada.

\section{Références}

1. Comité consultatif national de l'immunisation (CCNI). Mise à jour sur l'immunisation durant la grossesse avec le vaccin combiné anti-Tétanos, et à dose réduite contre la diphtérie et la coqueluche acellulaire (dcaT) [Consulté le $1^{\mathrm{er}}$ mars 2018]. Agence de la santé publique du Canada. https://www.canada. $\mathrm{ca} / \mathrm{fr} /$ sante-publique/services/publications/vie-saine/mise-jou r-immunisation-durant-grossesse-avec-vaccin-dcat.html

2. Comité consultatif national de l'immunisation (CCNI). Revue documentaire sur l'immunisation durant la grossesse avec le vaccin combiné anti-Tétanos, et à dose réduite contre la diphtérie et la coqueluche acellulaire (dcaT) : Innocuité, immunogénicité et efficacité [Consulté le $1^{\text {er }}$ mars 2018]. Agence de la santé publique du Canada. https://www. canada.ca/fr/sante-publique/services/publications/ vie-saine/sommaire-revue-documentaire-mise-jour-immuni sation-durant-grossesse-avec-vaccin-dcat.html 